2 DR AMY CHUNG (Orcid ID : 0000-0003-0020-9704)

3 MS ALANA WHITCOMBE (Orcid ID : 0000-0002-1951-3469)

4 DR NICOLE J MORELAND (Orcid ID : 0000-0001-6548-637X)

5

6

Article type : Outstanding Observation

Systems Immunology reveals a linked IgG3-C4 response in patients with

\title{
Acute Rheumatic Fever
}

Amy W Chung ${ }^{1}$, Timothy $\mathrm{KC} \mathrm{Ho}^{2}$, Paulina Hanson-Manful ${ }^{2,3}$, Susanne

Tritscheller ${ }^{2}$, Jeremy R Raynes ${ }^{2,3}$, Alana W Whitcombe ${ }^{2,3}$, Mei Lin Tay ${ }^{2,3}$, Reuben

McGregor $^{2,3}$, Natalie Lorenz ${ }^{2,3}$, Jane R Oliver ${ }^{1,4}$, Jason K Gurney ${ }^{4}$, Cristin G Print ${ }^{2,3}$, Nigel J

Wilson ${ }^{5}$, William J Martin ${ }^{6}$, Deborah A Williamson ${ }^{1}$, Michael G Baker ${ }^{4}$ and Nicole J

Moreland $2,3 *$

${ }^{1}$ Peter Doherty Institute for Infection and Immunity, University of Melbourne, Melbourne,

Victoria, Australia, ${ }^{2}$ School of Medical Sciences, University of Auckland, New Zealand,

${ }^{3}$ Maurice Wilkins Centre for Biodiscovery, University of Auckland, New Zealand,

${ }^{4}$ University of Otago, Wellington, New Zealand, ${ }^{5}$ Starship Children's Hospital, Auckland,

New Zealand, ${ }^{6}$ Callaghan Innovation, Wellington, New Zealand

*Correspondence to NJ Moreland (n.moreland@auckland.ac.nz)

Running title: Complement and immunoglobulin in rheumatic fever

Key words: rheumatic fever, complement, immunoglobulin, C4, IgG3, M protein

\section{Abstract}

This is the author manuscript accepted for publication and has undergone full peer review but has not been through the copyediting, typesetting, pagination and proofreading process, which may lead to differences between this version and the Version of Record. Please cite this article as doi: $10.1111 /$ IMCB.12298

This article is protected by copyright. All rights reserved 
31 Acute rheumatic fever (ARF) and chronic rheumatic heart disease (RHD) are autoimmune sequelae of a Group A Streptococcus (GAS) infection with significant global mortality and poorly understood pathogenesis. Immunoglobulin and complement deposition were observed in ARF/RHD valve tissue over 50 years ago, yet contemporary investigations have been lacking. This study applied systems immunology to investigate the relationships between the complement system and immunoglobulin in ARF. Patients were stratified by C-Reactive Protein (CRP) concentration into high $\left(\geq 10 \mu \mathrm{gL}^{-1}\right)$ and low $\left(<10 \mu \mathrm{g} \mathrm{mL}^{-1}\right)$ groups to distinguish those with clinically significant inflammatory processes from those with abating inflammation. The circulating concentration of 17 complement factors and six immunoglobulin isotypes and subclasses were measured in ARF patients and highly matched healthy controls using multiplex bead-based immunoassays. An integrative statistical approach combining feature selection (LASSO) and principal component analysis revealed a linked IgG3-C4 response in ARF patients with high CRP that was absent in controls. Strikingly, both IgG3 and C4 were elevated above clinical reference ranges, suggesting these features are a marker of ARF associated inflammation. Humoral immunity in response to $\mathrm{M}$ protein, an antigen implicated in ARF pathogenesis, was completely polarised to IgG3 in the patient group. Furthermore, the anti-M protein IgG3 response was highly correlated with circulating IgG3 concentration, highlighting a potential role for this potent immunoglobulin subclass in disease. In conclusion, a linked IgG3-C4 response appears important in the initial, inflammatory stage of ARF and may have immediate utility as a clinical biomarker given the lack of specific diagnostic tests currently available.

\section{Word count: 248}

\section{INTRODUCTION}

Acute rheumatic fever (ARF) is a severe post-infectious consequence of a Group A streptococcal (GAS) infection that can result in rheumatic heart disease (RHD). ARF is an acute inflammatory illness in which patients present with fever, migratory polyarthritis, subcutaneous nodules and carditis ${ }^{1}$. Recurrences of ARF with carditis can lead to permanent heart valve damage and RHD ${ }^{2}$. Unlike ARF where active inflammation contributes to symptoms, RHD is post-inflammatory and chronic in nature ${ }^{3}$. Globally there are approximately 33 million people living with RHD and this condition is responsible for over 300,000 deaths per annum ${ }^{4}$. ARF and RHD are generally considered diseases of the developing world, yet they persist in indigenous populations in the developed world. Māori 
and Pacific children in New Zealand and Aboriginal children in Australia have among the highest incidences of ARF globally 5,6.

ARF pathogenesis is poorly understood with the current prevailing hypothesis based on the notion that the alpha helical coiled-coil structure of GAS M-protein mimics human proteins with a coiled-coiled structure ${ }^{1}$. This mimicry is thought to induce M-protein specific antibodies and T-cells that cross-react with cardiac myosin, laminin and tropomyosin in the heart and synovium 7. However, the role of mimicry is debated, with an alternative hypothesis suggesting that GAS colonisation results in disruption of the extracellular matrix and exposure of cryptic collagen epitopes, which trigger an autoimmune response ${ }^{8-10}$.

Despite the current uncertainty around triggering antigen(s), the humoral basis for ARF was documented more than five decades ago when Kaplan et al. observed immunoglobulin and complement factor $\mathrm{C} 3$ deposits on the heart valves of children who had died from ARF with RHD ${ }^{11}$. The presence of $\mathrm{C} 3$ in these inflamed valves suggests a role for complement in ARF pathogenesis, yet contemporary analyses of the complement system in ARF are lacking. Technological advancements now enable the quantification and/or function of large panels of serum proteins to be determined concurrently in multiplex assays ${ }^{12,13}$. When combined with systems level computational approaches that allow for multidimensional analysis, the data generated by these high throughput assays can identify novel combinations of immune features associated with a particular disease state or treatment response ${ }^{14-16}$. The aim of this study was to apply these technologies to enable the first systematic and comprehensive analysis of the relationships between the complement system and immunoglobulin in ARF. The circulating concentration of complement factors that span the initiation (classical, lectin and alternative pathways), amplification and effector phases of the complement cascade, together with six immunoglobulin isotypes and subclasses in a cohort of ARF patients and matched healthy controls is reported. By applying systems serology data modelling techniques ${ }^{15}$, a unique complement-immunoglobulin relationship was revealed that provides new insight into ARF inflammation.

\section{RESULTS}

\section{Demographics and stratification by concentration of C-reactive Protein}

The 69 participants comprised children diagnosed with ARF according to the New Zealand modification of the Jones criteria $17 \quad(n=34)$ and matched healthy controls $(n=35)$. Demographics are shown in Table 1. Single serum samples were obtained from each ARF 
participant during their hospital stay and the C-reactive protein (CRP) concentration was used to stratify patients into 'high' ( $\geq 10 \mu \mathrm{g} \mathrm{mL} \mathrm{m}^{-1}$, clinically significant inflammation) and 'low' CRP $\left(<10 \mu \mathrm{g} \mathrm{mL}^{-1}\right.$, reduced inflammation) groups ${ }^{18}$. All 69 participants identified as either Māori or Pacific and the median age across the three groups was 11-11.5 years old, reflecting the peak incidence age for ARF in New Zealand ${ }^{19}$.

\section{Circulating complement factors and immunoglobulin}

The concentration of the 17 complement factors, 6 different immunoglobin isotypes and subclasses, and anti-C1q were determined using bead-based immunoassays and ELISA methodologies and compared between the ARF-high and ARF-low CRP groups and healthy controls in a single variable manner. Seven of the 24 analytes were significantly elevated $(P<0.05$, mean FC $>1.5)$ in the ARF-high CRP group compared with healthy controls, with levels trending back to control levels in the ARF-low CRP group (Figure 1, Table S1). This included the opsonization pre-cursor $\mathrm{C} 4$, complement system regulators factor $\mathrm{B}$ and $\mathrm{H}$, and the immunoglobulins IgG1, IgG3, IgG4 and IgA. The clear asymmetry of the Volcano plot for healthy controls compared with the ARF-high CRP group (Figure 1b) illustrates a general trend for elevation of circulating complement factors and immunoglobulin in ARF patients. Indeed, no notable reductions in the circulating concentrations of any analyte were observed in the ARF patient groups compared with the healthy controls, though the concentrations of some (including anti-C1q antibodies, the alternative pathway regulator Factor D, and the effector phase component C9) were essentially unchanged. When clinical reference ranges were applied to the seven significantly elevated analytes, only IgG3 and C4 showed marked elevation in the ARF-high CRP group; $18 / 20$ patients (90\%) and 10/20 patients (50\%) had IgG3 and $\mathrm{C} 4$ concentrations above the upper limit of the reference range, respectively (Figure 1c). These proportions were reduced in the ARF-low CRP group such that for IgG3 11/14 patients (78\%) and for $\mathrm{C} 44 / 14$ patients (28\%) had concentrations above references ranges. For the remaining five analytes, $\leq 25 \%$ of patients in the ARF-high CRP group had serum concentrations above clinically accepted normal ranges.

\section{Identification of Rheumatic Fever Specific Features}

To further explore relationships between complement and immunoglobin in ARF, and to identify key features contributing to differences between groups, "systems immunology" methodology was applied ${ }^{15,20}$. Multidimensional analysis combining feature selection (the 
127 least absolute shrinkage and selection operator; LASSO) and principal component analysis

128 (PCA) were used to analyze the 27 features comprising the 24 analytes from the univariate 129 analysis together with age, gender and CRP concentration. Four of the 27 features accounted 130 for $82 \%$ of the variance between healthy controls and patients in the ARF-high CRP group, 131 when assessing across two PCA dimensions (Figure 2). Near to complete separation of the 132 healthy controls from ARF-high CRP patients is shown in the scores plot, with each point 133 representing individual healthy controls (blue) or ARF (yellow) participants (Figure 2c). The 134 first dimension (PC1) accounted for most of the variance between the groups (64.2\%), and 135 the corresponding loadings plot (Figure 2d) shows the contribution of the four features (C4, $136 \mathrm{IgG} 3, \operatorname{IgA}$ and $\mathrm{IgG1})$ selected by LASSO. The scores plot highlights some heterogeneity 137 amongst ARF patients with the second dimension (PC2) accounting for $17.8 \%$ of the 138 variance (Figure 2c). However, the majority of individuals in the ARF group (65\%) are 139 skewed towards elevated $\mathrm{C} 4$ and $\mathrm{IgG} 3$ and scatter with these features in multidimensional 140 space (Figure 2d).

141 When the three groups were analyzed simultaneously (healthy controls, ARF-high CRP and 142 ARF-low CRP), the same four features separated the participant profiles and accounted for $14376.5 \%$ of the variance (Supplementary Figure 1). Again, the first dimension (PC1) accounted 144 for most of the variance (59.3\%), with the scores and loading plots highlighting a dominant effect of $\mathrm{IgG} 3$ and $\mathrm{C} 4$ on the rheumatic fever profile.

146 The proximity of IgG3 and C4 in the loadings plots prompted a correlation analysis of these 147 two features. Scatter plots show that the concentration of serum IgG3 and C4 were 148 significantly correlated in the ARF-high CRP group $(P<0.05)$ but not in healthy controls 149 (Figure 3).

\section{Antigen Specific Humoral Responses in Rheumatic Fever}

151 To determine whether the increase in circulating IgG3 observed in the ARF-high CRP group 152 was due to IgG3 specific for GAS antigens associated with ARF, ELISA detecting the four 153 IgG isotypes were performed. M protein was chosen as an ARF associated antigen since 154 antibodies specific for M protein repeats are proposed to cross-react with cardiac antigens 155 and cause valvulitis ${ }^{1,21}$. The M6 protein was selected as a representative M protein as emm6 156 strains have been associated with ARF in New Zealand ${ }^{22}$. As a comparator, the conserved 157 GAS antigen Streptolysin-O (SLO) was chosen as it is known to be highly immunogenic but 158 is not implicated in ARF pathogenesis ${ }^{23,24}$. 
159 A marked difference in the IgG subclass response to the two GAS antigens was observed in 160 the ARF-high CRP participants. For SLO, IgG1 was the most abundant subclass, with IgG2, $161 \mathrm{IgG} 3$ and IgG4 detected at moderate levels. In contrast, the response induced by M6 was 162 dominated by $\mathrm{IgG} 3$, followed by $\mathrm{IgG} 1$, with $\mathrm{IgG} 2$ and $\mathrm{IgG} 4$ responses low or undetectable 163 (Figure $4 \mathrm{a}$ ). When the ratio of $\operatorname{IgG} 3 / \operatorname{IgG1}$ was plotted there was a highly significant 164 difference between SLO and M6 $(P<0.0001)$, such that for all 20 ARF patients $(100 \%)$ the 165 most abundant anti-M6 subclass was IgG3, while the most abundant anti-SLO subclass was 166 IgG1 (Figure 4b). Six of the 34 healthy control participants had evidence of a prior GAS exposure via clinical streptococcal serology ${ }^{23}$. Comparison of the $\operatorname{IgG}$ subclass response in these six participants with the ARF-high CRP patients showed reduced M6 IgG3 polarization (Supplementary Figure 2). Indeed, only 3/6 (50\%) healthy controls had IgG3 as the most abundant anti-M6 subclass and the ratio of anti-M6 IgG3/IgG1 in the ARF-high participants was significantly elevated compared with controls $(P<0.01)$.

172 To investigate whether the M6 anti-IgG3 in ARF patients was directed towards the 173 hypervariable (HVR) N-terminus of the M protein, or the more conserved repeat regions implicated in pathogenesis 7,25 , ELISA were performed with a 50-mer peptide corresponding to the M6 HVR sequence. Four of the 20 participants in the ARF-high CRP group had elevated total IgG to M6 HVR (Supplementary Figure 3), indicative of a previous infection with an M6 GAS strain ${ }^{26}$. Further ELISA in these four M6-positive ARF patients showed the type-specific, HVR response was dominated by IgG1, in contrast with the IgG3 response for the full-length M6 protein (Supplementary Figure 3). Thus, it can be assumed that most of the IgG3 M protein binding in the ARF-high CRP group is to the more conserved repeat regions of the protein.

182 Finally, a correlation analysis performed between the circulating levels of IgG3 with antigen specific isotype responses found a significant correlation between anti-M6 IgG3 levels and total IgG3 $(P<0.05)$, but not anti-SLO IgG3 levels and total IgG3 (Figure 4c). This suggests anti-M protein antibodies may contribute to the elevated IgG3 observed in ARF patient sera.

\section{DISCUSSION}

187 This study has examined the circulating levels of complement factors and immunoglobulin in children with ARF utilizing sera obtained during hospital admission. A striking elevation of IgG3 and $\mathrm{C} 4$ was observed in ARF patients with clinically significant inflammation $\left(\mathrm{CRP} \geq 10 \mu \mathrm{g} \mathrm{mL}^{-1}\right)$ with $90 \%$ and $50 \%$, respectively, having circulating concentrations above reference ranges. Though other immunoglobulins and complement factors were 
elevated, $\leq 25 \%$ of patients had circulating concentrations above reference ranges suggesting these analytes maybe of less clinical importance. The application of a multivariate, systems serology approach to analyse the data set also identified IgG3 and C4 as key features associated with ARF. This, together with a significant correlation between the circulating concentration of $\mathrm{IgG} 3$ and $\mathrm{C} 4$ suggests an important role for a linked IgG3-C4 response in ARF inflammation.

The association of IgG3, the most potent IgG subclass with respect to classical pathway activation ${ }^{27}$, and $\mathrm{C} 4$, an early classical pathway component, might suggest classical pathway driven complement activation in ARF inflammation. This is supported by studies conducted over 40 years ago that found IgG (total), C3 and C4 were elevated in circulation, yet decreased in the synovial fluid of affected joints in ARF patients ${ }^{28,29}$. In the early stages of disease, IgG3-C4 complement driven inflammation may contribute to immune activation at affected tissue sites in ARF. This process appears to reverse as inflammation abates given the concentration of complement and immunoglobulin features in patients with low CRP return to control levels, and decreases in complement and immunoglobulin were observed in a recent proteomic study of post-inflammatory RHD ${ }^{30}$.

An acute phase response to proinflammatory cytokines, rather than activation of the complement cascade per se, may also contribute to $\mathrm{C} 4$ elevation. Complement $\mathrm{C} 3$ and $\mathrm{C} 4$ are well described acute phase reactants - that is plasma proteins which increase in response to inflammatory stimulus and proinflammatory cytokines such as interleukin(IL)-6, IL-1, tumor necrosis factor- $\alpha$ and interferon(IFN)- $\gamma^{31}$. Secretion of both IL-6 and IFN- $\gamma$ is significantly elevated in peripheral blood mononuclear cells from ARF patients stimulated with GAS ${ }^{32}$ and circulating concentrations of proinflammatory cytokines are elevated in ARF (reviewed by ${ }^{33}$ ). Thus, the linked IgG3-C4 response observed here may be a marker for a proinflammatory state in ARF patients.

The significant elevation in complement $\mathrm{C} 4$ in ARF contrasts with the reduction in $\mathrm{C} 4$ associated with post-streptococcal glomerulonephritis (PSGN), the other major postinfectious sequalae associated with GAS infection ${ }^{33}$. Indeed, transient depression of $\mathrm{C} 1 \mathrm{q}$, C2, C3 and C4 are hallmarks of PSGN ${ }^{34,35}$ and are thought to result from immune complex formation with complement consumption, and lead to glomerular immune deposits ${ }^{34}$. The opposing observation of increased complement features in ARF suggests very different roles for complement in these two GAS sequelae. Understanding the proposed contributions of (i) IgG3 mediated activation of the classical complement cascade, versus (ii) proinflammatory 
cytokines and acute phase responses, to the linked IgG3-C4 response in ARF will require broader interrogation of immune features. Yet, regardless of the contributing mechanisms, IgG3 and C4 may have immediate clinical utility as disease biomarkers. Specific diagnostic tests for ARF are lacking ${ }^{10}$, and the incorporation of $\operatorname{IgG} 3$ and $\mathrm{C} 4$ into the panel of tests currently utilized may aid differential diagnosis, particularly as levels were elevated above reference ranges.

A central question in ARF pathogenesis is the role of M-protein specific antibody cross-reactivity in causing disease ${ }^{8}$. In this study the humoral response to M6 protein was found to be dominated by IgG3, whereas the response to SLO, a conserved antigen not implicated in ARF pathogenesis, was dominated by IgG1. Polarization to an IgG3 humoral response to $\mathrm{M}$ protein was previously observed in a study of patients with GAS associated sore throats, cellulitis and glomerulonephritis ${ }^{36}$. However, the IgG3 predominance was not as marked with $48 \%$ of the sera tested having an IgG3:IgG1 ratio $>1.0$, compared with $100 \%$ of ARF participants in this study. A recent investigation of adaptive immunity to two conserved GAS antigens in adults compared with children found polarization to IgG3 increases with age ${ }^{37}$. It is possible that in ARF, there is dysregulation of the antigen specific IgG3 response at a younger age due to repeated exposure to GAS in at risk populations. This process of "immune priming" 38 may contribute to the significant elevation of M protein specific IgG3 observed in this study. Given the diversity of M types epidemiologically associated with ARF ${ }^{22}$, and that the hypervariable N-terminus of the M6 protein induced an IgG1 dominated response, it is likely that most of the observed IgG3 $\mathrm{M}$ protein binding is to the more conserved B-and $\mathrm{C}$ - repeat regions of the protein. While these $\mathrm{M}$ protein regions have been proposed to contain cross-reactive epitopes to host proteins ${ }^{1}$, it is difficult to postulate on any possible link to pathogenesis in the absence of a confirmed and clinically validated target for autoantibodies in $\mathrm{ARF}^{39}$. Nevertheless, the elevation of $\mathrm{M}$ protein specific IgG3 in ARF may be a consideration for GAS vaccine development, where the need to generate protective immune responses should be balanced with the potential risks of eliciting a harmful response. In conclusion, this study has utilized multiplex assays combined with multidimensional analysis to identify a linked IgG3-C4 response in patients with ARF. This response may be a marker for patients in a proinflammatory state and, as IgG3 and C4 are elevated above normal reference ranges, may have utility in ARF diagnostics.

\section{METHODS}




\section{Study subjects}

258 Human blood samples were obtained from participants recruited as part of the Rheumatic Fever Risk Factors (RF RISK) study conducted in New Zealand between 2014-2017. All participants (or proxies) provided written informed consent and the protocols had appropriate ethical board approval (HDEC 14/NTA/53). This study included sera from participants recruited in the first 18 months (September 2014 - April 2016) of a three-year recruitment period. Patients with first episode ARF were diagnosed according to the New Zealand modification of the Jones criteria ${ }^{17}$ and those with a 'probable/definite' diagnosis were included, while those with categorised as 'suspect ARF' were excluded. Control samples were obtained from healthy participants that were matched for age, ethnic identification, area-based measures of sociodemographic deprivation (as assessed by the New Zealand Deprivation Index score ${ }^{40}$ ) and geographic area (district health board region in which the subject resides). Blood specimens were collected into serum clotting tubes and serum was separated by centrifugation, frozen and stored at $-80{ }^{\circ} \mathrm{C}$ until analysis.

\section{Measurement of C-reactive protein}

272

The CRP concentration in sera was determined using a custom made singleplex cytometric bead array assay (BioLegend LEGENDplex, San Diego) as per the manufacturer's instructions. Serum samples were diluted 1:600 and fluorescence was detected using a flow cytometer (BD Accuri C6). Data analysis was performed using Flow Cytometric Analysis Program (FCAP) Array software, version 3 (Becton-Dickinson, New Jersey).

277 Given the varied length of hospital admission and broad range of disease severity associated with ARF presentation, patients were stratified into those with CRP $<10 \mu \mathrm{g} \mathrm{mL}^{-1}$ (ARF-low $\mathrm{CRP}$ ) and $\mathrm{CRP} \geq 10 \mu \mathrm{g} \mathrm{mL} \mathrm{mL}^{-1}$ (ARF-high CRP). As an acute phase protein, CRP is released during systemic inflammation and tissue damage ${ }^{41}$ and was used to distinguish participants with clinically significant inflammatory processes from those in which these processes were abating ${ }^{18}$. Healthy controls with $\mathrm{CRP} \geq 10 \mu \mathrm{g} \mathrm{mL}-1$ were excluded from further analysis since, in the absence of full clinical information, it was difficult to interpret whether elevated CRP was due to an undiagnosed infection or inflammatory condition or another, unrelated reason. Of the 45 healthy controls recruited in the 18 -month timeframe, 35 had CRP $<10 \mu \mathrm{g}$ $\mathrm{mL}^{-1}$ and were included in all subsequent analysis (Table 1). These 35 healthy controls were screened for prior GAS exposure via streptococcal serology using bead-based immunoassays as previously published ${ }^{23}$. 
290 The concentration of six different immunoglobulin isotypes and subclasses were measured in 291 the sera using magnetic human immunoglobulin isotyping beads (Merck Millipore,

292 Darmstadt). The assays were run in a 5-plex format containing beads for IgG1, IgG2, IgG4, $293 \operatorname{IgM}$ and IgA with a serum dilution of 1:16,000. A singleplex assay was needed for IgG3 that 294 required a serum dilution of 1:50,000 for the resulting Median Fluorescence Intensities (MFI) 295 to fall within the range of the standard curve. Assay plates were read using a Magpix 296 Analyser (Luminex Corporation, Austin) and MFI was converted to concentration using the associated Xponent software (version 4.2, Luminex Corporation, Austin).

\section{Complement System Factors}

299

Two different bead-based platforms were utilized to measure complement system factors.

The concentrations for $\mathrm{C} 3 \mathrm{a}$ and $\mathrm{C} 4 \mathrm{a}$ were determined by Cytometric Bead Array (CBA) assay (Human Anaphylatoxin Kit, Becton-Dickinson, New Jersey) using a serum dilution of 1:6000, while C5a was measured using the same kit and a dilution of 1:300. Fluorescence was detected using a flow cytometer (BD Accuri C6, New Jersey) and conversion to concentration was performed using Flow Cytometric Analysis Program (FCAP) Array software, version 3 (Becton-Dickinson, New Jersey). A further 15 complement factors were measured using human complement magnetic bead panels (Merck Millipore, Darmstadt). One panel included beads for C2, C4b, C5, C5a, C9, Factor D, Mannose-Binding lectin (MBL) and Factor I and required a serum dilution of 1:200, while the second panel included beads for C1q, C3, C3b, C4, Factor B, Factor $\mathrm{H}$ and Properdin and required a serum dilution of 1:40,000. Fluorescence was detected using a Magpix Analyser (Luminex Corporation, Austin) and MFI was converted to concentration using the associated Xponent software (version 4.2, Luminex Corporation, Austin). C5a was included in both the CBA and magnetic bead panels, with a strong correlation between the assays (Pearson $\mathrm{r} 0.86, P<0.001$ ) such that only data from the magnetic bead panel are presented.

315 The concentration of $\mathrm{C} 1 \mathrm{q}$ auto-antibodies in sera were measured using a $\mathrm{C} 1 \mathrm{q}$ antibody

316 Enzyme-Linked Immunosorbent Assay (ELISA) kit and a 1:100 dilution as per the 317 manufacturer's instructions (Abnova Corporation, Taipei City).

\section{IgG1 and IgG3 Enzyme-Linked Immunosorbent Assays}

319 The M6 and SLO proteins used in IgG isotype ELISA were prepared using standard 320 recombinant expression techniques in Escherichia coli. M6 protein from MGAS10394 was 
321 produced as a mature protein, with the signal sequence and transmembrane removed, via expression in BL21(DE3) pLysS E. coli followed by immobilized-metal affinity chromatography (IMAC) as previously described ${ }^{42}$. SLO from MGAS SF370 was produced without the signal sequence, and with $\mathrm{P} 427 \mathrm{~L}$ and $\mathrm{W} 535 \mathrm{~F}$ mutations to remove lysin activity, via expression in BL21-AI E. coli and IMAC purification as published ${ }^{43}$. The recombinant His $_{6}$-tag was cleaved from M6 and SLO using 3C and rTEV protease, respectively, and both antigens were further purified by gel filtration using a Superdex 200 column (GE Lifesciences, Pittsburgh) ${ }^{42}$. The M6 50-mer peptide corresponding to the N-terminal HVR sequence was obtained commercially (GenScript, Piscataway, New Jersey).

For ELISA using recombinant proteins, Nunc-immunoplates (Sigma-Aldrich, St Louis) were coated with antigen at $5 \mu \mathrm{g} \mathrm{ml}^{-1}$ and blocked with Phosphate Buffered Saline supplemented with $0.1 \%$ Tween-20 and 5\% skim milk powder for 1 hour at room temperature (RT). Serum was added at a 1:200 dilution and incubated for 1 hour at RT. IgG binding was detected using isotype specific secondary antibodies labelled with horse-radish peroxidase at 1:500 dilution as recommended by the manufacturer (ThermoFisher Scientific, Waltham). Antigen coating for ELISA using the M6 50-mer peptide was modified according to published protocols ${ }^{26}$. The resulting absorbance readings were converted to normalized ELISA Units (nEU) in GraphPad Prism (Version 8.0) using a 7-point standard curve constructed with positive control sera. The lowest dilution (1:500) on the standard curve was designated $10 \mathrm{nEU}$, while the highest dilution $(1: 1,039,500)$ was designated $0.01 \mathrm{nEU}$ and the same standard curve was included on each ELISA plate.

\section{Data analysis and statistics}

343 The univariate analysis of immunoglobulin and complement factors utilized a Kruskal-Wallis test for comparison between three groups (healthy control, high CRP cases and low CRP cases), with Dunn's multiple comparisons test. The IgG isotype ELISA were analysed using a Wilcoxon matched pairs signed rank test for comparison of antigen specific Ig in each sera sample. Correlations were calculated using Spearman r. All of these analyses were performed in GraphPad Prism (Version 8.0) and a $P$-value of $\leq 0.05$ was considered significant.

349 Key features that contributed to differences between groups were identified using principal component analysis (PCA) ${ }^{44}$, while least absolute shrinkage and selection operator (LASSO) penalized regression feature selection method ${ }^{45}$, was used to reduce the dimensions of the outcome variables. Cross-validation was performed iteratively to find the optimal value of the 
353

354

355

356

357

358

359

360

361

362

363

364

365

366

367

368

369

370

371

372

373

374

375

376

377

378

379

380

381

382

383

384

regularized parameters (k-fold cross validation). Prior to analysis, all data were normalized with mean centering and variance scaling.

\section{ACKNOWLEDGMENTS}

This work was funded by the Maurice Wilkins Centre for Biodiscovery and a Heart Foundation of New Zealand Senior fellowship to NJM. The RF RISK study, from which samples were obtained, was funded by the Heath Research Council of New Zealand (HRC) Rheumatic Fever Research Partnership (Ministry of Health, Te Puni Kōkiri, Cure Kids, Heart Foundation, and HRC). We thank staff in paediatric departments across the North Island of New Zealand for kind support and assistance. All members of the RF RISK Study (in addition to the authors on this manuscript) are gratefully acknowledged.

\section{REFERENCES}

1 Carapetis JR, Beaton A, Cunningham MW et al. Acute rheumatic fever and rheumatic heart disease. Nat Rev Dis Primers 2016; 1: 1-24.

2 Carapetis JR, McDonald M, Wilson NJ. Acute rheumatic fever. Lancet 2005; 366: 155168.

3 Steer AC. Historical aspects of rheumatic fever. Journal of Paediatrics and Child Health $2015 ; \mathbf{5 1}: 21-27$

4 Watkins DA, Johnson CO, Colquhoun SM et al. Global, Regional, and National Burden of Rheumatic Heart Disease, 1990-2015. N Engl J Med 2017; 377: 713-722.

5 Milne RJ, Lennon DR, Stewart JM, Vander Hoorn S, Scuffham PA. Incidence of acute rheumatic fever in New Zealand children and youth. Journal of Paediatrics and Child Health 2012; 48: 685-691.

6 Parnaby MG, Carapetis JR. Rheumatic fever in indigenous Australian children. Journal of Paediatrics and Child Health 2010; 46: 527-533.

7 Cunningham MW. Streptococcus and rheumatic fever. Current Opinion in Rheumatology 2012; 24: 408-416.

8 Tandon R, Sharma M, Chandrashekhar Y, Kotb M, Yacoub MH, Narula J. Revisiting the pathogenesis of rheumatic fever and carditis. Nat Rev Cardiol 2013; 10: 171-177.

9 Cunningham MW. Rheumatic fever revisited. Nat Rev Cardiol 2014; 11: 123.

10 Karthikeyan G, Guilherme L. Acute rheumatic fever. Lancet 2018; 392: 161-174. 
385

386

387

388

389

390

391

392

393

394

395

396

397

398

399

400

401

402

403

404

405

406

407

408

409

410

411

412

413

414

415

416

417

11 Kaplan MH, Bolande R, Rakita L, Blair J. Presence of bound immunoglobulins and complement in the myocardium in acute rheumatic fever. Association with cardiac failure. N Engl J Med 1964; 271: 637-645.

12 Bongoni AK, Lanz J, Rieben R, Banz Y. Development of a bead-based multiplex assay for the simultaneous detection of porcine inflammation markers using xMAP technology. Cytometry A 2013; 83: 636-647.

13 Lash GE, Pinto LA. Multiplex cytokine analysis technologies. Expert Rev Vaccines 2010; 9: 1231-1237.

14 Kidd BA, Peters LA, Schadt EE, Dudley JT. Unifying immunology with informatics and multiscale biology. Nat Immunol 2014; 15: 118-127.

15 Chung AW, Alter G. Systems serology: profiling vaccine induced humoral immunity against HIV. Retrovirology 2017; 14: 57.

16 Nakaya HI, Pulendran B. Vaccinology in the era of high-throughput biology. Phil Trans $R$ Soc B 2015, doi:10.1086/650310.

17 Atatoa-Carr P, Lennon D, Wilson N, New Zealand Rheumatic Fever Guidelines Writing Group. Rheumatic fever diagnosis, management, and secondary prevention: a New Zealand guideline. $N Z$ Med J 2008; 121: 59-69.

18 Kushner I, Antonelli MJ. What should we regard as an 'elevated' C-reactive protein level? Ann Intern Med 2015; 163: 326.

19 Gurney JK, Stanley J, Baker MG, Wilson NJ, Sarfati D. Estimating the risk of acute rheumatic fever in New Zealand by age, ethnicity and deprivation. Epidemiol Infect $2016 ; 1-10$.

20 Chung AW, Kumar MP, Arnold KB et al. Dissecting Polyclonal Vaccine-Induced Humoral Immunity against HIV Using Systems Serology. Cell 2015; 163: 988-998.

21 Fischetti VA. Streptococcal M protein: molecular design and biological behavior. Clin Microbiol Rev 1989; 2: 285-314.

22 Williamson DA, Smeesters PR, Steer AC et al. M-Protein Analysis of Streptococcus pyogenes Isolates Associated with Acute Rheumatic Fever in New Zealand. J Clin Microbiol 2015; 53: 3618-3620.

23 Hanson-Manful P, Whitcombe AL, Young PG et al. The novel Group A Streptococcus antigen SpnA combined with bead-based immunoassay technology improves streptococcal serology for the diagnosis of acute rheumatic fever. J Infect 2018; 76: 361368. 
418

419

420

421

422

423

424

425

426

427

428

429

430

431

432

433

434

435

436

437

438

439

440

441

442

443

444

445

446

447

448

449

450

451

24 Bensi G, Mora M, Tuscano G et al. Multi High-Throughput Approach for Highly Selective Identification of Vaccine Candidates: the Group A Streptococcus Case. Molecular \& Cellular Proteomics 2012; 11: M111.015693-M111.015693.

25 Smeesters PR, McMillan DJ, Sriprakash KS. The streptococcal M protein: a highly versatile molecule. Trends in Microbiology 2010; 18: 275-282.

26 Frost HR, Laho D, Sanderson-Smith ML et al. Immune Cross-Opsonization Within emm Clusters Following Group A Streptococcus Skin Infection: Broadening the Scope of Type-Specific Immunity. Clin Infect Dis 2017; 65: 1523-1531.

27 Damelang T, Rogerson SJ, Kent SJ, Chung AW. Role of IgG3 in Infectious Diseases. Trends Immunol 2019; 40: 197-211.

28 Bhattacharya S, Reddy KS, Sundaram KR et al. Differentiation of patients with rheumatic fever from those with inactive rheumatic heart disease using the artificial subcutaneous nodule test, myocardial reactive antibodies, serum immunoglobulin and serum complement levels. Int J Cardiol 1987; 14: 71-78.

29 Svartman M, Potter EV, Poon-King T, Earle DP. Immunoglobulins and complement components in synovial fluid of patients with acute rheumatic fever. J Clin Invest 1975; 56: $111-117$.

30 Mukherjee S, Jagadeeshaprasad MG, Banerjee T et al. Proteomic analysis of human plasma in chronic rheumatic mitral stenosis reveals proteins involved in the complement and coagulation cascade. Clinical Proteomics 2014; 11: 35.

31 Markanday A. Acute Phase Reactants in Infections: Evidence-Based Review and a Guide for Clinicians. Open Forum Infect Dis 2015; 2: ofv098.

32 Kim ML, Martin WJ, Minigo G et al. Dysregulated IL-1 $\beta-G M-C S F$ Axis in Acute Rheumatic Fever That Is Limited by Hydroxychloroquine. Circulation 2018; 138: 26482661.

33 Martin WJ, Steer AC, Smeesters PR et al. Post-infectious group A streptococcal autoimmune syndromes and the heart. Autoimmunity Reviews 2015; 14: 710-725.

34 Balasubramanian R, Marks SD. Post-infectious glomerulonephritis. Paediatr Int Child Health 2017; 37: 240-247.

35 Eison TM, Ault BH, Jones DP, Chesney RW, Wyatt RJ. Post-streptococcal acute glomerulonephritis in children: clinical features and pathogenesis. Pediatr Nephrol 2011; 26: $165-180$.

36 Falconer AE, Carson R, Johnstone R, Bird P, Kehoe M, Calvert JE. Distinct IgG1 and IgG3 subclass responses to two streptococcal protein antigens in man: analysis of 
452

453

454

455

456

457

458

459

460

461

462

463

464

465

466

467

468

469

470

471

472

473

474

475

476

477

antibodies to streptolysin $\mathrm{O}$ and $\mathrm{M}$ protein using standardized subclass-specific enzymelinked immunosorbent assays. Immunology 1993; 79: 89-94.

37 Mortensen R, Nissen TN, Blauenfeldt T, Christensen JP, Andersen P, Dietrich J. Adaptive Immunity against Streptococcus pyogenes in Adults Involves Increased IFN- $\gamma$ and IgG3 Responses Compared with Children. The Journal of Immunology 2015; 195: $1657-1664$.

38 Raynes JM, Frost HRC, Williamson DA et al. Serological Evidence of Immune Priming by Group A Streptococci in Patients with Acute Rheumatic Fever. Front Microbiol 2016; 7: 1119

39 Bright PD, Mayosi BM, Martin WJ. An immunological perspective on rheumatic heart disease pathogenesis: more questions than answers. Heart 2016; 102: 1527-1532.

40 Salmond C, Crampton P, King P, Waldegrave C. NZiDep: a New Zealand index of socioeconomic deprivation for individuals. Soc Sci Med 2006; 62: 1474-1485.

41 Clos Du TW, Mold C. C-reactive protein: an activator of innate immunity and a modulator of adaptive immunity. Immunol Res 2004; 30: 261-277.

42 Jones S, Moreland NJ, Zancolli M et al. Development of an opsonophagocytic killing assay for group a streptococcus. Vaccine 2018; 36: 3756-3763.

43 Chiarot E, Faralla C, Chiappini N et al. Targeted amino acid substitutions impair streptolysin O toxicity and group A Streptococcus virulence. MBio 2013; 4: e00387-12.

44 Jolliffe IT. Principal Component Analysis. Springer Series in Statistics. Springer, New York, NY, 1986.

45 Tibshirani R. The lasso method for variable selection in the Cox model. Stat Med 1997; 16: $385-395$.

Table 1. Demographic characteristics and C-reactive protein concentration of study participants

\begin{tabular}{|c|c|c|c|}
\hline Characteristic & $\begin{array}{l}\text { Healthy control* } \\
\qquad n=35\end{array}$ & $\begin{array}{c}\text { ARF High } \\
\left(\mathrm{CRP} \geq 10 \mu \mathrm{g} \mathrm{mL}^{-1}\right) \\
\mathrm{n}=\mathbf{2 0}\end{array}$ & $\begin{array}{c}\text { ARF Low } \\
\left(\text { CRP }<10 \mu \mathrm{g} \mathrm{mL}^{-1}\right) \\
\mathrm{n}=14\end{array}$ \\
\hline $\begin{array}{l}\text { Age, median (IQR), } \\
\text { years }\end{array}$ & $11.0(8.0-13.0)$ & $11.5(9.3-13.0)$ & $11.0(9.0-13.0)$ \\
\hline Male sex (\%) & $23(66)$ & $13(65)$ & $7(50)$ \\
\hline
\end{tabular}




\begin{tabular}{lccc}
\hline Māori (\%) & $13(37)$ & $7(35)$ & $3(21)$ \\
Pacific (\%) & $22(63)$ & $13(65)$ & $11(79)$ \\
CRP concentration & & & \\
$\left(\mu \mathbf{g ~ m L} \mathbf{~ m}^{-1}\right)$ & & & \\
Mean [95\% CI] & $2.17[1.46-2.88]$ & $49.44[22.16-76.72]$ & $5.94[4.43-7.45]$ \\
Median [95\% CI] & $1.38[0.85-2.23]$ & $23.78[17.12-53.70]$ & $6.41[3.18-8.37]$ \\
\hline
\end{tabular}

* Participants recruited into the control arm with $\mathrm{CRP} \geq 10 \mu \mathrm{g} \mathrm{mL}^{-1}$ were excluded $(\mathrm{n}=10)$

FIGURE LEGENDS

480

481

Figure 1. Circulating complement factors and immunoglobulin. (a) Heatmap showing the concentration of analytes in ARF-high $(n=20)$ and ARF-low CRP $(n=14)$ groups compared to healthy controls $(n=35)$. Data represent two technical replicates. Fold change (FC) was determined from the means of each group and $P$-value by Kruskal-Wallis one-way analysis of variance. FC and $P$-value are indicated by coloured boxes and analytes with $\mathrm{FC}>1.5$ and $P$ $<0.05$ are marked with a triangle. (b) Volcano plot comparing the concentration of analytes in healthy controls with ARF-high CRP patients. FC was determined from the means and $P$ value by Dunn's multiple comparisons test. Dotted lines indicate FC of 1.5 and $P$-value of 0.05 , and analytes with $\mathrm{FC}>1.5$ and $P<0.05$ are shown in red. (c) Serum concentration of Ig (mg mL $\mathrm{m}^{-1}$ ) and complement factors $\left(\mu \mathrm{g} \mathrm{mL}^{-1}\right)$ in the healthy control (HC), ARF-high CRP (RF-H) and ARF-low CRP (RF-L) groups. Bars represent mean $\pm \mathrm{SD}$ and the $P$-values were determined by Dunn's multiple comparisons test. The upper limit of clinical reference ranges are shown as dashed lines.

Figure 2. PCA and LASSO Identify Rheumatic Fever Specific Features.

(a and b) (a) Scores plot for the PCA represents the ARF-high CRP (RF-H; yellow, n=20) and healthy controls (HC; blue, $\mathrm{n}=35$ ) for each participant tested (dots). (b) Features included in the PCA analysis. PC1 and PC2 captured $38.97 \%$ of the variance. (c and d) (c), PCA and LASSO for the ARF-high CRP (yellow, n=20) and healthy controls (blue, $\mathrm{n}=35$ ). (d) The four features listed on the loadings plot accounted for $82 \%$ of the variance between the two groups. See also Supplementary Figure S1.

Figure 3. The linked IgG3-C4 response in Rheumatic fever. Scatter plots comparing the circulating concentration of total IgG3 and C4 in the ARF-high CRP group (RF-H; yellow, 
$505 \mathrm{n}=20)$ and healthy controls (HC; blue, $\mathrm{n}=35)$. Data represent two technical replicates.

506 Spearman $\mathrm{r}$ and $P$-values as shown.

507

508

509 Figure 4. IgG responses in Acute Rheumatic Fever Patients. (a) IgG subclasses specific 510 for M6 (grey) and SLO (white) in the ARF-high CRP group ( $\mathrm{n}=20)$ as measured by ELISA. 511 Data are shown as normalized ELISA units (nEU) and bars represent the median nEU for 512 each IgG isotype. (b) Ratio of IgG3:IgG1 in individual sera for the ARF-high CRP group to 513 M6 and SLO proteins. For (a and b), $P$-values were determined by Wilcoxon matched pairs 514 signed rank test and data are representative of three independent experiments. (c) Scatter 515 plots comparing the circulating concentration of total IgG3 with the IgG3 response to M6 and 516 SLO in the ARF-high CRP group. Spearman $\mathrm{r}$ and $P$-values as shown. See also 517 Supplementary Figure S2 and S3.

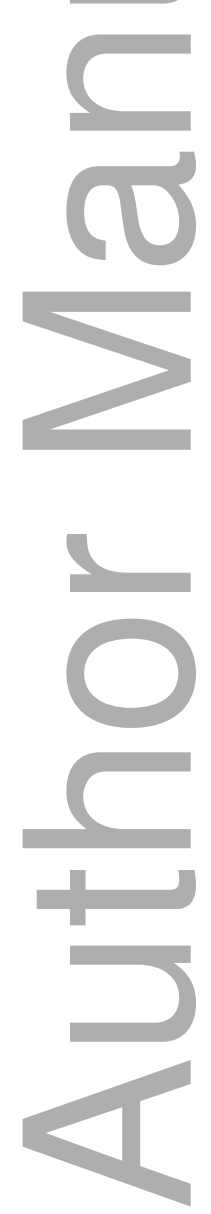


(a)

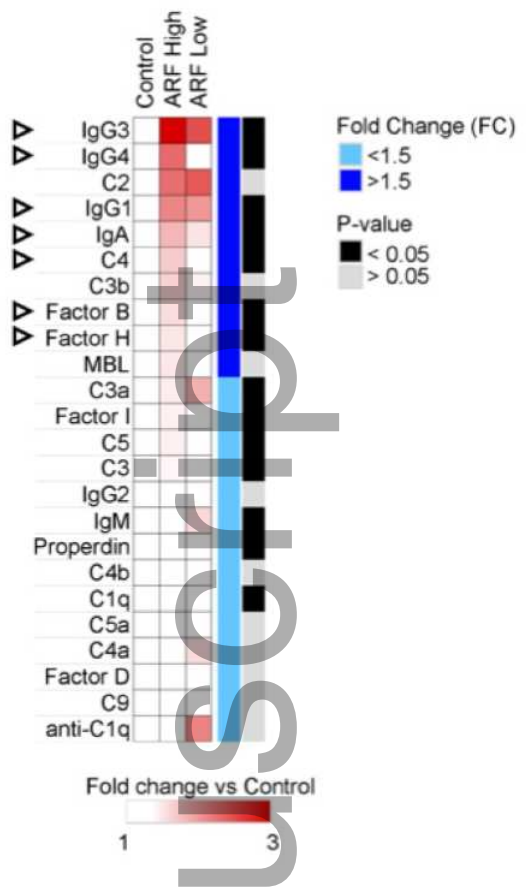

(b)

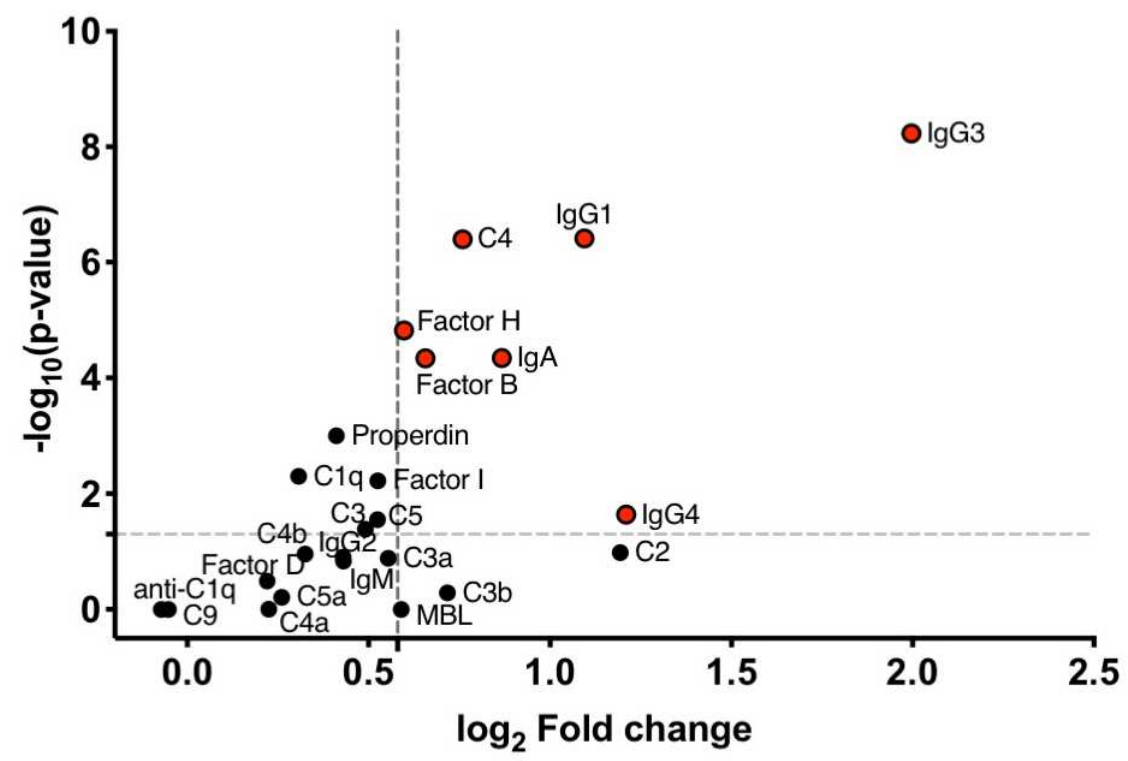

(c)
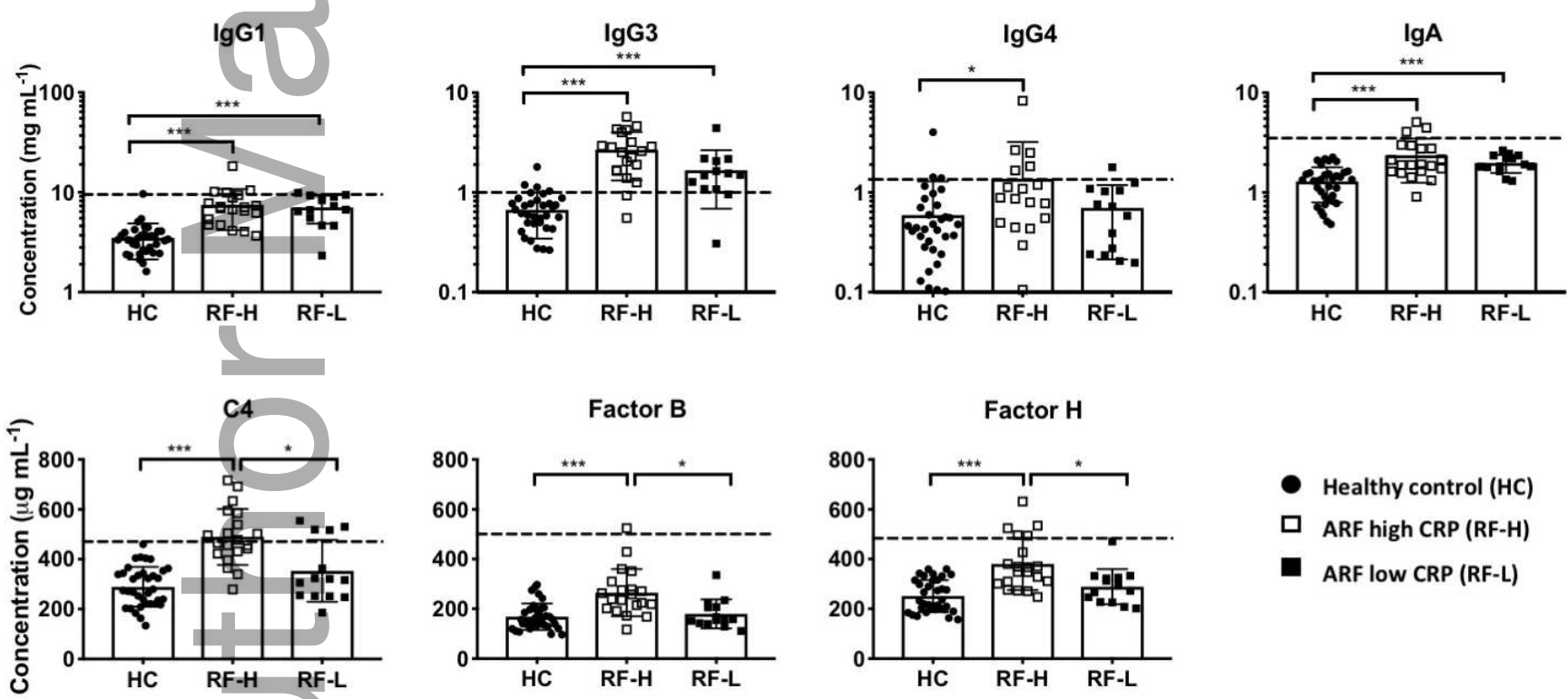

- Healthy control (HC)

a ARF high CRP (RF-H)

ARF low CRP (RF-L)

imcb_12298_f1.jpg 
(a)

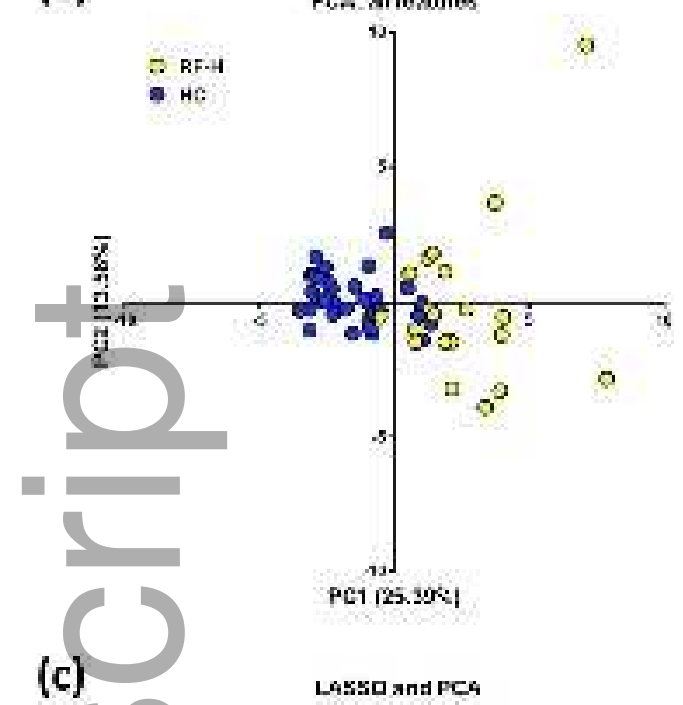

(b)

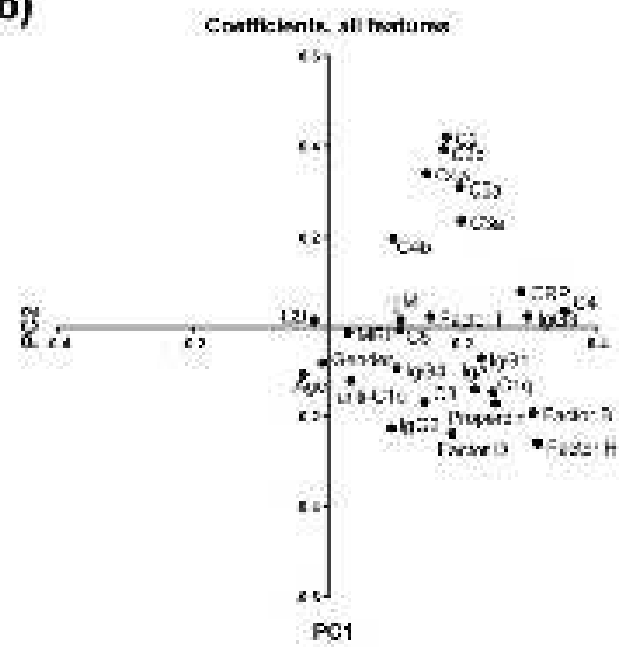

(d)

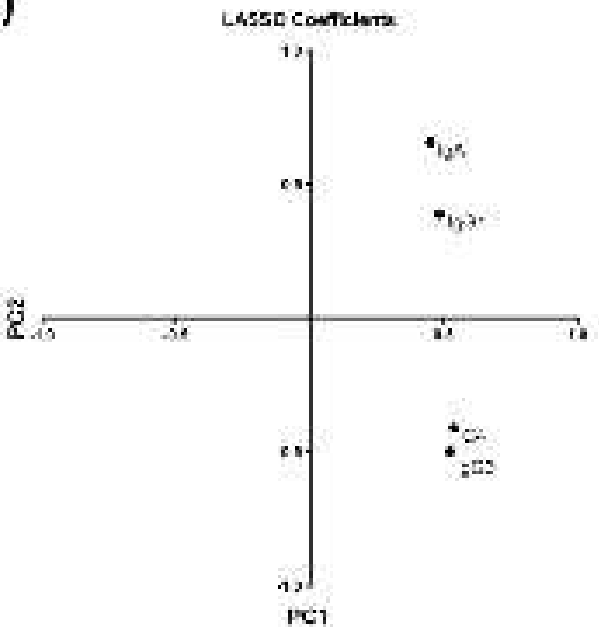

imcb_12298_f2.jpeg

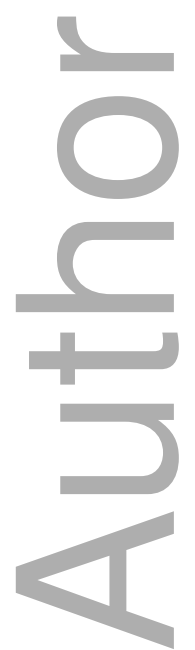



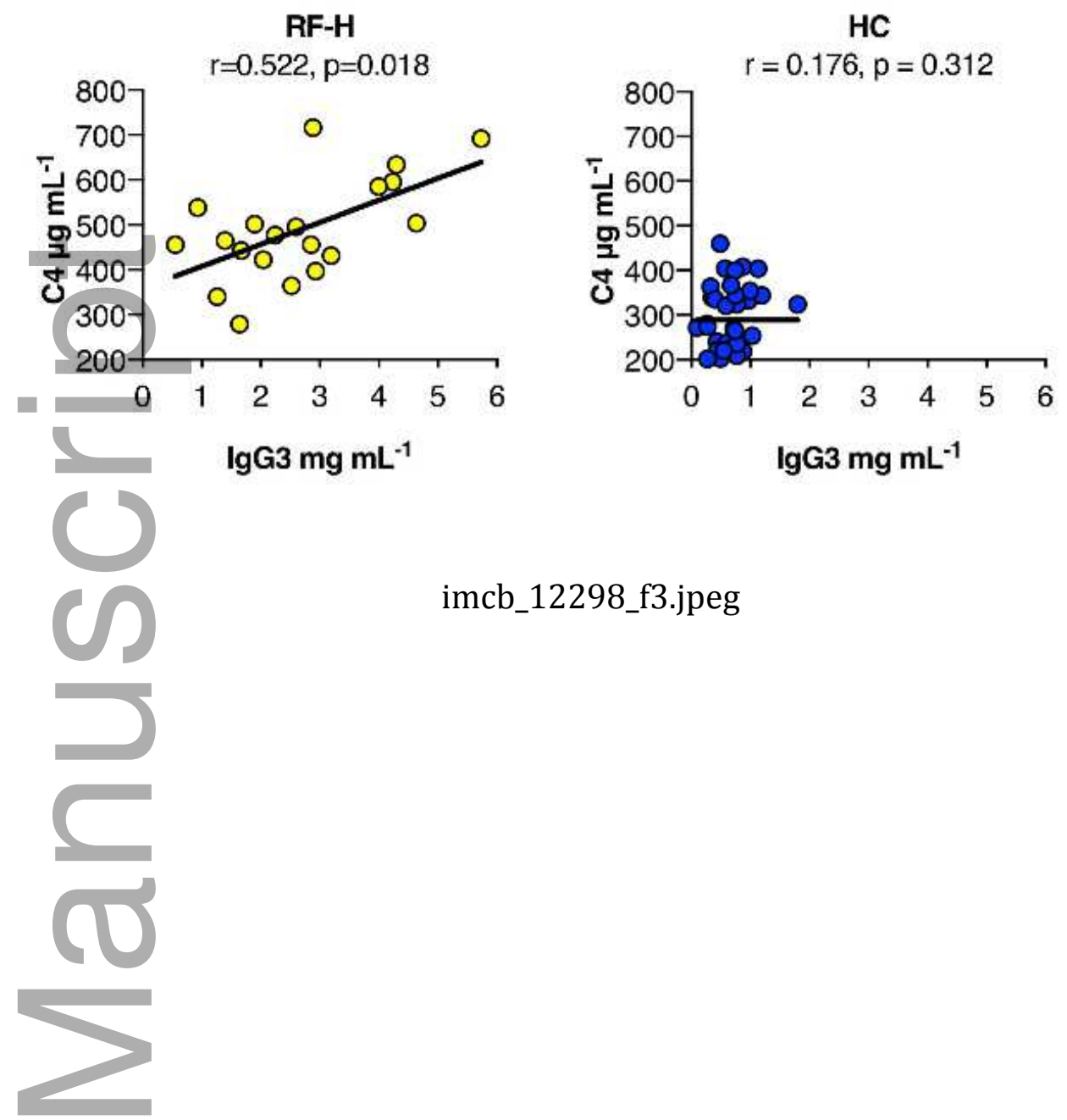

imcb_12298_f3.jpeg

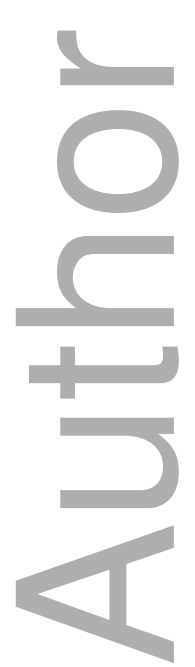

This article is protected by copyright. All rights reserved 
(a)

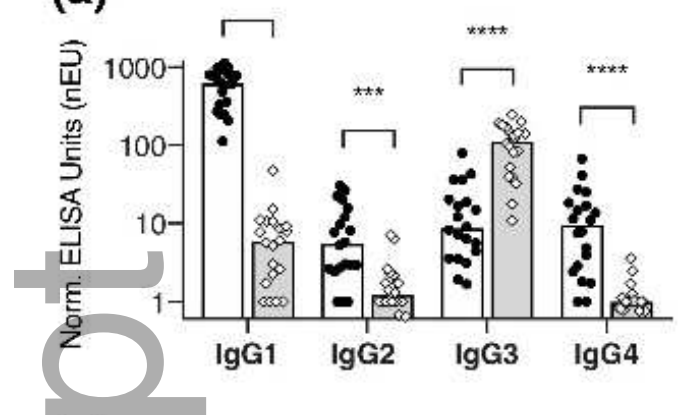

(b)

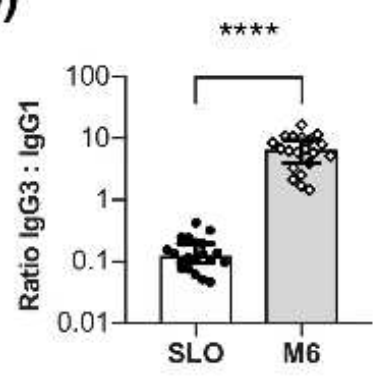

(c)

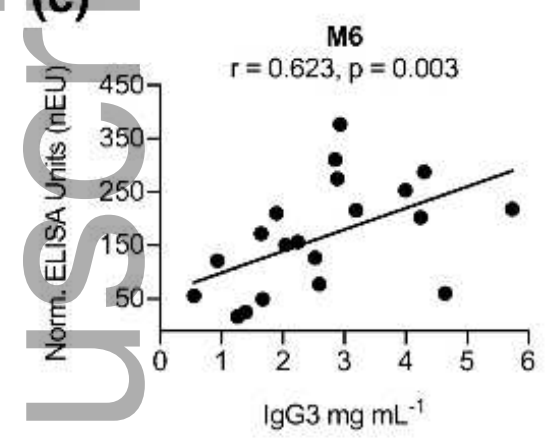

- SLO

M6

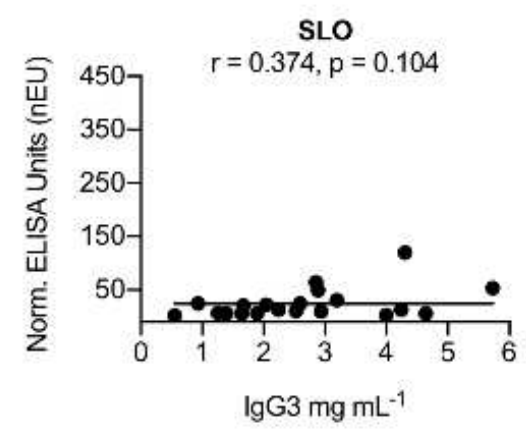

imcb_12298_f4.jpg
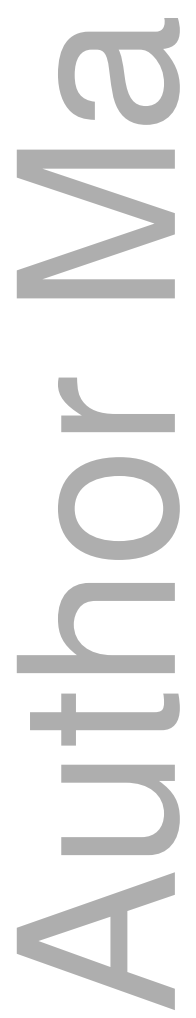

This article is protected by copyright. All rights reserved 


\section{University Library}

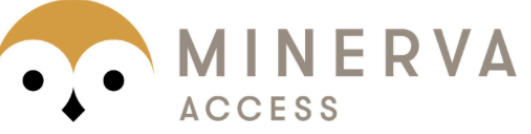

A gateway to Melbourne's research publications

Minerva Access is the Institutional Repository of The University of Melbourne

\section{Author/s:}

Chung, AW;Ho, TKC;Hanson-Manful, P;Tritscheller, S;Raynes, JM;Whitcombe, AL;Tay, ML;McGregor, R;Lorenz, N;Oliver, JR;Gurney, JK;Print, CG;Wilson, NJ;Martin, WJ;Williamson, DA;Baker, MG;Moreland, NJ

Title:

Systems immunology reveals a linked IgG3-C4 response in patients with acute rheumatic fever

\section{Date:}

2019-11-18

\section{Citation:}

Chung, A. W., Ho, T. K. C., Hanson-Manful, P., Tritscheller, S., Raynes, J. M., Whitcombe, A. L., Tay, M. L., McGregor, R., Lorenz, N., Oliver, J. R., Gurney, J. K., Print, C. G., Wilson, N. J., Martin, W. J., Williamson, D. A., Baker, M. G. \& Moreland, N. J. (2019). Systems immunology reveals a linked IgG3-C4 response in patients with acute rheumatic fever. IMMUNOLOGY AND CELL BIOLOGY, 98 (1), pp.12-21. https://doi.org/10.1111/imcb.12298.

Persistent Link:

http://hdl.handle.net/11343/286628 